Research Article

\title{
Optimization of Magnetic-Grating-Like Stroke-Sensing Cylinder Based on Response Quality Evaluation Algorithm
}

\author{
Yanqing Guo,, ${ }^{1,2}$ Lu Liu, ${ }^{2}$ Yongling Fu $\mathbb{D}^{,},{ }^{3}$ Chuangchuang $\mathrm{Li}^{2}$ and Liang Guo ${ }^{2}$ \\ ${ }^{1}$ Shanxi Key Laboratory of Advanced Manufacturing Technology, North University of China, No. 3 Xueyuan Road, \\ Jiancaoping District, Taiyuan, Shanxi, China \\ ${ }^{2}$ School of Mechanical and Power Engineering, North University of China, No. 3 Xueyuan Road, Jiancaoping District, Taiyuan, \\ Shanxi, China \\ ${ }^{3}$ School of Mechanical Engineering and Automation, Beihang University, No. 37 Xueyuan Road, Haidian District, Beijing, China \\ Correspondence should be addressed to Yongling Fu; fuyongling@126.com
}

Received 28 May 2017; Revised 5 October 2017; Accepted 6 November 2017; Published 11 March 2018

Academic Editor: Jesus Corres

Copyright (C) 2018 Yanqing Guo et al. This is an open access article distributed under the Creative Commons Attribution License, which permits unrestricted use, distribution, and reproduction in any medium, provided the original work is properly cited.

\begin{abstract}
The measurement of hydraulic cylinder displacement has been addressed from different fields. The detection principle of magnetic grating is able to realize the high integration and accuracy. In this paper, a signal response quality evaluation algorithm for devising and optimizing a high-accuracy displacement measuring system is proposed. On the basic of signal response quality evaluation method, structure variables are optimized to enhance the working performance. By defining the parameters, an optimum structure cylinder prototype is made and tested to provide better estimates. Experimental results on working characteristic are presented to verify the effectiveness of the optimized structure. The efficiency of the proposed signal response quality evaluation function is therefore demonstrated through the working performance.
\end{abstract}

\section{Introduction}

In the past decades, the development of electro-hydraulic actuators (EHA) allows flight-control systems to balance requirements of high power, lightweight, safety, fast response, and continuity of service [1]. Actuation system has introduced to meet the control surface loading and response demands. The Airbus A380 employs EHA in its flight surface control system as a state-of-the-art example for exclusion of complex hydraulic lines from controlling systems [2]. In other words, the architecture of EHA is sufficiently robust to failures so as to ensure flight safety and minimize the size of the control system [3]. Within the design procedure, considering system integration, miniaturization, and measuring precision, the displacement measuring methodology of hydraulic cylinders is one of the most fundamental and promising research settings in the field of EHA.

Studies emerged have paved a way for research on cylinder displacement sensing methods of EHA. In the industrial practice, two kinds of position sensing devices are used: one is linear variable inductor displacement transducer (LVDT), and the other is magnetostrictive displacement sensor $[4,5]$. The resolution and accuracy of such a sensor are mainly determined by measuring principle and working conditions. Since the distribution of actuation system requires high integration under a severe working environment, both measurements are difficult for installation and maintenance due to their large volumes and complex structures. In such cases, it is advisable to introduce a more appropriate method for cylinder stroke measurement.

Previously, Murakami and Kitsunai applied the idea of equipping the hydraulic cylinder with a stroke sensor based on a magnetoresistor sensor to detect positions [6]. Their results indicated that a high magnetic field and a large field length are required for sensing devices working. Compared with the approaches mentioned above, magneticgrating-like stroke-sensing cylinder, which is also known as magnetic-grating-like hydraulic cylinder integrated displacement sensor, provides a more effective alternative. This sensing device is to use simple working elements that will 


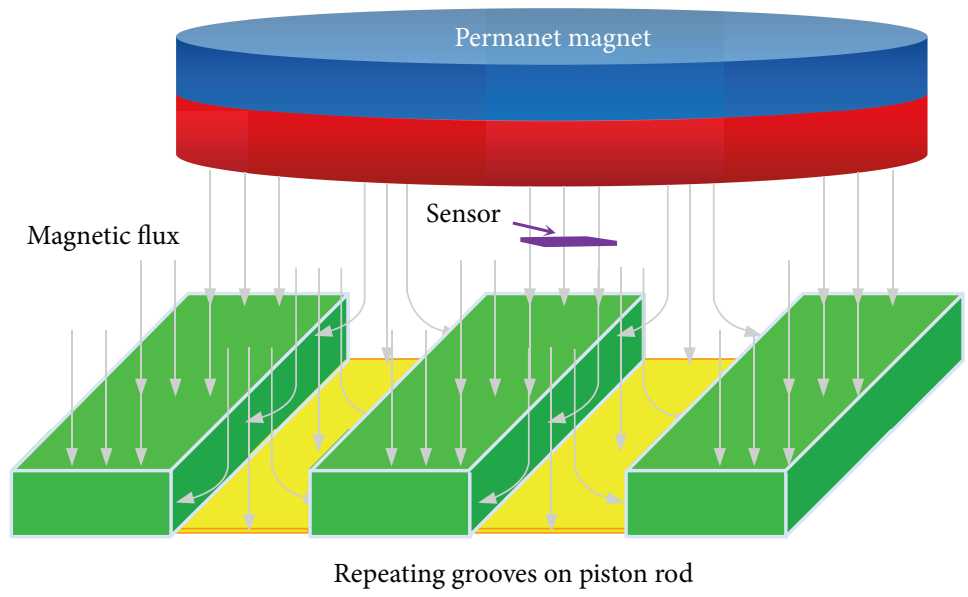

FIGURE 1: Structure of magnetic-grating-like stroke-sensing cylinder.

minimize the size of EHA by minimizing the dimension of the cylinder [7]. With magnetic-grating-like stroke-sensing cylinder, a combination of cylinder and sensor can be designed to handle long working distance measurement as well as matching with the structure of EHA. On the other hand, Yang et al. proposed a stroke-sensing cylinder based on Hall sensors associated with the automation of heavy construction machinery, which reduced the effect of noise with specific electric circuits [8]. The magnetic-grating-like stroke-sensing cylinder investigated in this research is composed of a piston rod, permanent magnet, and a Hall sensor, as shown in Figure 1.

The permanent magnet generates magnetic fields while the sensor detects the magnetic field intensity and thereby measures the displacement. The piston rod is made of ferromagnetic materials with the repeating grooves on the body. The intensity variation is sampled as a function of the relative position between the permanent magnet and the piston rod by positioning the receiving magnetic force across a range of lateral offsets. When the piston rod is moving, the permanent magnet forms constant magnetic scales on the piston rod in combination with the repeating grooves. The magnetic induction line is thus periodically modulated. Supposing the piston rod is moving with a fixed speed, the induction line along with the sensor is simulated, as shown in Figure 2.

The sensor can effectively detect the magnetic field depending on the magnetic scale, in which the displacement is measured in a magnetic-grating sensing way. The Hall sensor is employed to sense signals on the cylinder's surface with magnetic scale [9]. The Hall elements measure the variation of the magnetic flux density which is produced by the slots grooved on the surface of the cylinder. The sensing outcome of Hall sensor is likely to be harmonic signal within the working period (Figure 3). The signal glitch is because of the discrete block given through modeling and simulation, which stands for the dimensional precision of working elements.

The problem of magnetic-grating-like stroke-sensing cylinder employment arises in precision measurement, since current laboratory research is still not able to address this issue. Specifically, with the added sliding mode control, Yang et al. did not give the emphasis on accuracy improvement.
Most of the previous research aimed at setting up a preliminary system for sensing the stroke of the cylinder. To the best of our knowledge, specific structure design and optimization have not yet been established and no quantitative information is given.

In this paper, we will consider the reason for the low accuracy of magnetic-grating-like hydraulic cylinder integrated displacement sensor. A set of structural optimization method is proposed for addressing the design of the sensor. The research contributions of this paper can be summarized as follows:

(a) Response quantification criteria setup: the study develops a function which combined the signal subdivision principle and sensor output used for evaluating the measurement accuracy.

(b) Structural parameter optimization: define the structural variables by referring to the response quantification function and compare the influence of each component on optimization results.

(c) Performance testing design: to prove the technical efficacy of proposed optimization framework, the prototype is produced and experimental platform is designed. Through the characteristic testing, the measurement accuracy of magnetic-grating-like stroke-sensing cylinder can be obtained.

This paper will introduce signal subdivision-related knowledge and present specific response quality assessment method in Section 2, illustrate parameter optimization process and the optimal structure in Section 3, show the performance testing results achieved of experimental prototype and analyze the measurement accuracy in Section 4, and present future research expectation in Section 5.

\section{Response Quality Evaluation Algorithm}

2.1. Signal Subdivision Principle. The fundamental principle of stroke measurement is the evaluation of the relative displacement. When the piston rod moves with the distance 


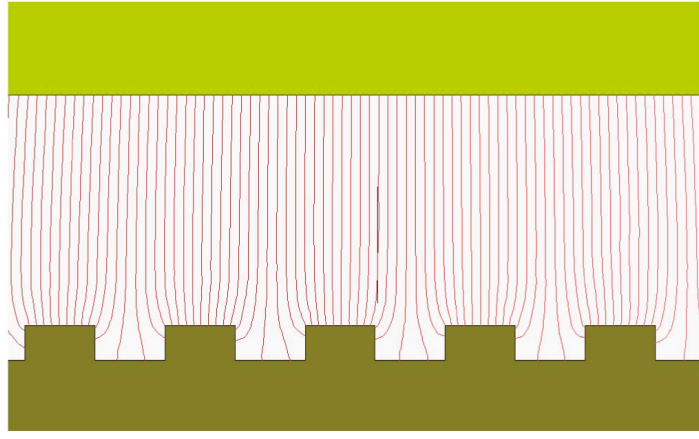

(a) At the position of $0 \mathrm{~mm}$

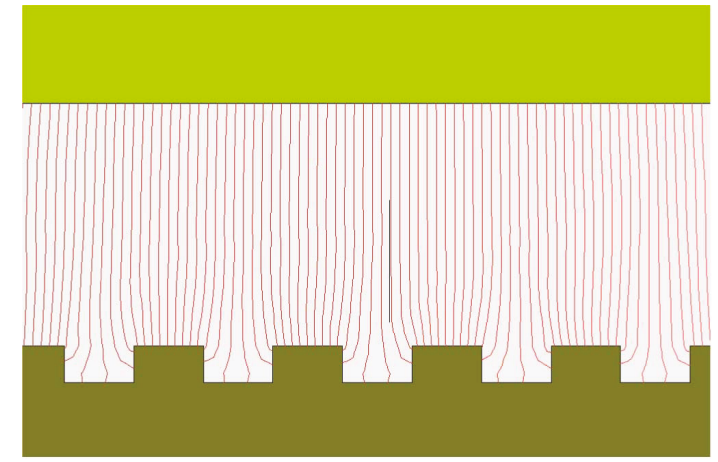

(b) At the position of $0.4 \mathrm{~mm}$

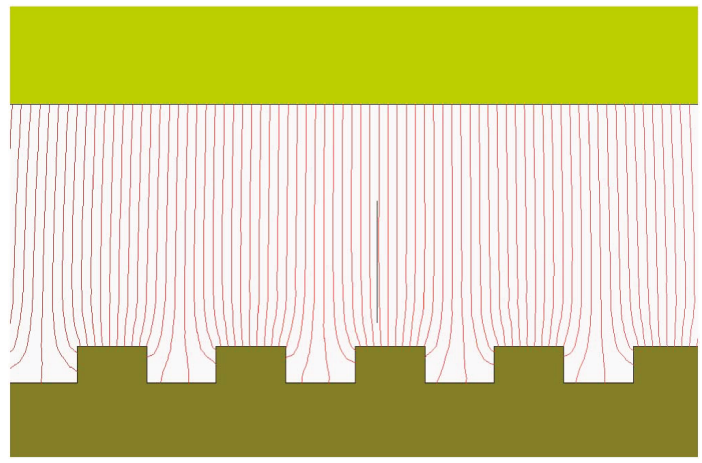

(c) At the position of $0.8 \mathrm{~mm}$

Figure 2: Periodic change of magnetic field intensity during the piston rod moving.

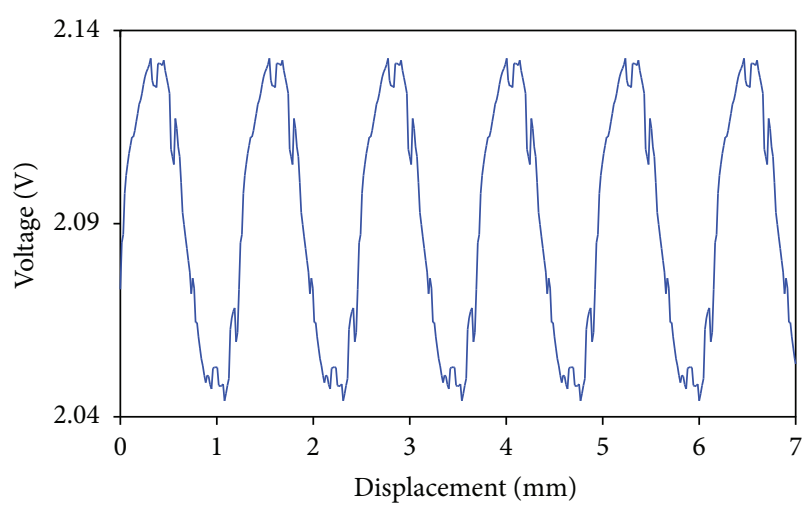

FIGURE 3: Curve of Hall sensor response by simulation.

of one slot, one complete period of harmonic signal will therefore be generated. The signal, which is sensed by the Hall sensor, reflects the variation of magnet field intensity. In this way, the measurement accuracy means the length of one-slot range. According to previous study, the responses of the grating sensor are sine and cosine signals [10]. Hence, the outcome can be normalized by the transformed circumferential angle from $0^{\circ}$ to $360^{\circ}$ in all cases. Suppose that the minimal length value to be measured is defined by unorganized angles. To start the accurate measurement process, a subdivision $0^{\circ}$ to $360^{\circ}$ should be generated from every single angle to attain a higher accuracy. Although the magnetic-grating-like stroke-sensing cylinder is inherently less sensitive to changes in displacement, the angle- segmented approach of the harmonic signal offers the advantage that only the response of the sensor is required to demodulate displacement from measured piston rod. In precision measurement, the angle of harmonic signal can be segmented into a far smaller angle change, based on the basic theory of Moiré Fringe signal subdivision technology $[11,12]$. At every given angle, the increment to the next is a controllable fixed amount, which can be regulated by the subdivision method. An analysis of the working performance evaluation criteria is presented in this section. If the position of cylinder within one slot is a standard harmonic signal, the testing outcome can be converted to the corresponding segmentation angle. The smaller the angle is divided, the higher precision can be acquired.

2.2. Tangent Method for Signal Subdivision. Lot of research has been conducted to address signal subdivision for performance criteria. Tangent-cotangent subdivision technique is a state-of-the-art method for grating signal segmentation according to Moiré Fringe electric subdivision technique $[13,14]$. The fundamental principle of tangent-cotangent subdivision is to investigate the tangent and cotangent value of the two orthogonal signals of different angles, when the output is reflected from a harmonic signal, that is,

$$
f_{\mathrm{tct}}(\theta)= \begin{cases}\left|\frac{A \sin \theta}{A \cos \theta}\right|, & \left|\frac{A \sin \theta}{A \cos \theta}\right| \leq 1, \\ \left|\frac{A \cos \theta}{A \sin \theta}\right|, & \left|\frac{A \cos \theta}{A \sin \theta}\right| \leq 1 .\end{cases}
$$




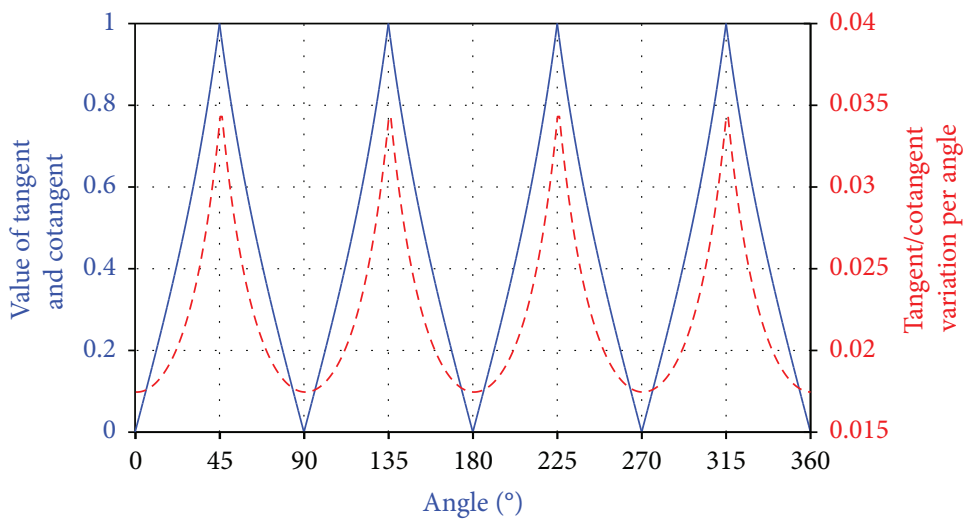

FIGURE 4: Tangent/cotangent variation for angle increments.

For simplicity, the expression is written in terms of angle, rather than radian.

In this way, the displacement can be calculated with the angle $\theta$ got by arctangent algorithm, which eliminates the low precision due to amplitude measurement. Considering the demand of high resolution, the angles will be segmented into even smaller division values. Aiming at establishing the response quantification criteria, the theoretical output of sensor can be calculated in advance. Based on the variation of structure, the response to system input varies. Thus, a function for evaluating the signal response should be set up for system optimization. The response $Q_{T}$ is

$$
Q_{T}=\max \left\{\left|f_{\mathrm{tct}}\left(\theta_{T_{-} i}\right)-f_{\mathrm{tct}}\left(\theta_{s_{-} i}\right)\right|\right\},
$$

where $\theta_{T i}$ is the angle of optimized modeling structure and $\theta_{s_{-} i}$ is the theoretical output angle corresponding to the displacement of piston rod. Accordingly, the maximum error between $\theta_{T_{-} i}$ and $\theta_{s_{-} i}$ stands for the quality of response. Due to the nonlinearity of the response $Q_{T}$, the resolution of (2) will inevitably bring large error to the result. Distinct unit increments of tangent and cotangent value range within the whole cycle (Figure 4).

In line with Figure 2, the change of tangent/cotangent is defined as a nonlinear function according to the angle increments from $0^{\circ}$ to $360^{\circ}$, which cannot be resolved by (2). However, the angle transformation period of objective function $Q_{T}$ is from $0^{\circ}$ to $90^{\circ}$, where the output can be mathematically revised for calculation. Further, two parts of the curve, $0-45^{\circ}$ and $45^{\circ}-90^{\circ}$, are symmetric to each other along the $y$-axis. Thus, the tangent/cotangent increment of $45^{\circ}-90^{\circ}$ is defined as

$$
\begin{aligned}
f_{\mathrm{Ad}}\left(\theta_{s_{-} i}\right)= & 2.3144 \times 10^{-16} \cdot \theta_{s_{-} i}{ }^{5}+1.8473 \times 10^{-9} \cdot \theta_{s_{-} i}{ }^{4} \\
& +6.6875 \times 10^{-7} \cdot \theta_{s_{-} i}{ }^{3}+9.5517 \times 10^{-5} \cdot \theta_{s_{-} i}{ }^{2} \\
& -0.0063 \cdot \theta_{s_{-} i}+0.1802
\end{aligned}
$$

where $\theta_{s_{-} i}$ ranges from $45^{\circ}$ to $90^{\circ}$.

$$
\text { Minimize } \min \left(f_{\mathrm{Ad}}\left(\theta_{s_{-} i}\right)\right)=0.017455 .
$$

During the parameter modification process, the variable $f_{\text {Ad }}\left(\theta_{s_{-} i}\right)$ is revised according to parameter distribution and it determines the quality of response within its angle range domain. $f_{\mathrm{Ad}}\left(\theta_{s_{-} i}\right)$ of the whole range is taking the cyclic variation of angle into account.

$$
\begin{aligned}
f_{\text {Ad }}\left(\theta_{s_{-} i}\right)= & 2.3144 \times 10^{-16} \cdot \mathrm{Ang}^{5}+1.8473 \times 10^{-9} \cdot \mathrm{Ang}^{4} \\
& +6.6875 \times 10^{-7} \cdot \mathrm{Ang}^{3}+9.5517 \times 10^{-5} \cdot \mathrm{Ang}^{2} \\
& -0.0063 \cdot \mathrm{Ang}+0.1802
\end{aligned}
$$

s.t. $\operatorname{Ang}=\left(\theta_{s_{-} i}-45\right) \bmod (90)+45$,

where mod represents taking the remainder of target values. The combined objective is defined by

$$
Q_{T}=\max \left\{\frac{\left|f_{\mathrm{tct}}\left(\theta_{T_{-} i}\right)-f_{\mathrm{tct}}\left(\theta_{s_{-} i}\right)\right| \times 0.017455}{f_{\mathrm{Ad}}\left(\theta_{s_{-} i}\right)}\right\} .
$$

We refer readers to [13-16] for more detailed information on Moiré Fringe electric subdivision and its application.

In order to verify the correctness of the adopted response quality evaluation method, we compute the error of predefined samples with its numerical change in combination with sin/cosine signal. In this test, white noise is added into the sinusoidal signal and cosine signal (Figure 5(a)). The shape of outcome angle error is defined by response evaluation function $Q_{T}$. Thus, the white noise error can be extracted with the evaluation function. The error response of different signals is given in Figures 5(b) and 5(c). As shown in Figure 4, the real deviation and calculating error can be distinguished only at the points of $45^{\circ}, 90^{\circ}, 135^{\circ}, 225^{\circ}$, and $315^{\circ}$. After the modification, the identification of white noise error is substantially improved in Figure 5(c).

For the purpose of structure optimization, the algorithm is implemented as the following steps:

Step 1. Take one working period of a specific sensor as the research sample.

Step 2. On the basic of signal subdivision principle, set the sensing response as harmonic signal.

Step 3. Divide the period into even parts which correspond to angle data and compute the theoretical tangent/cotangent value based on (1). 


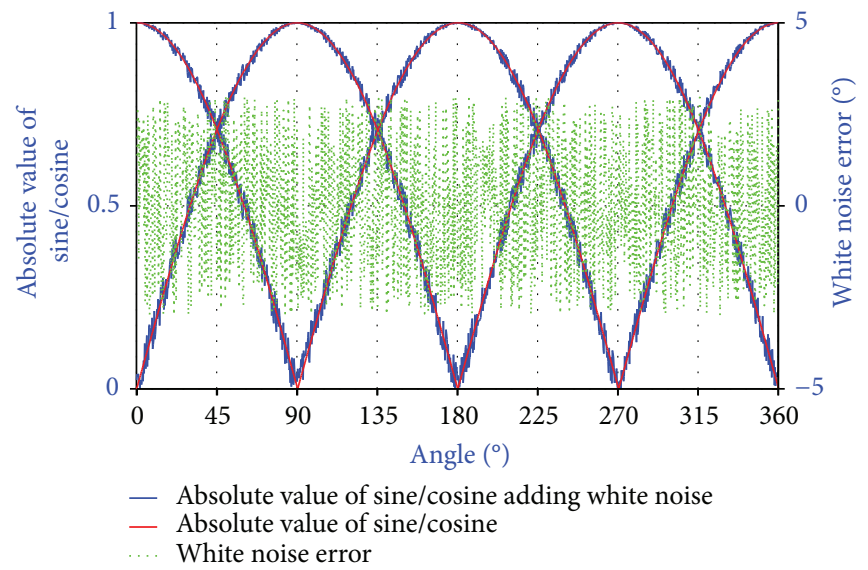

(a)

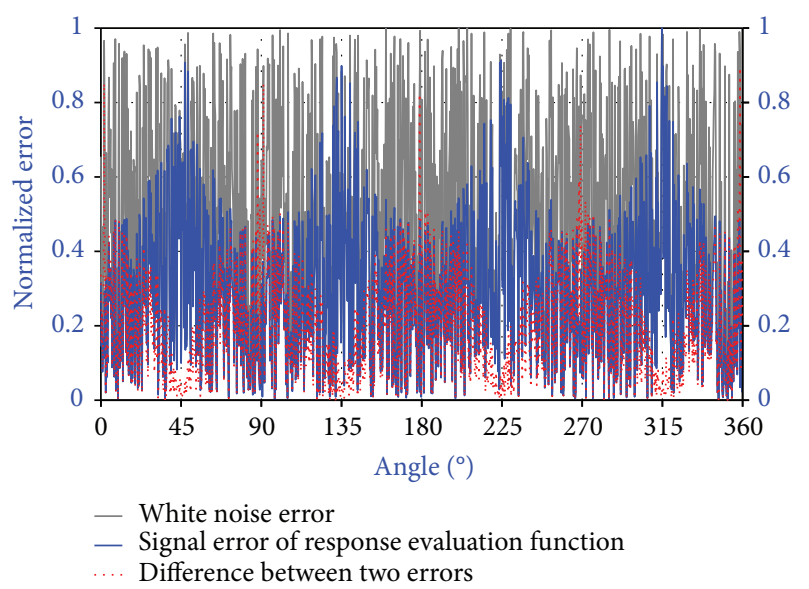

(b)

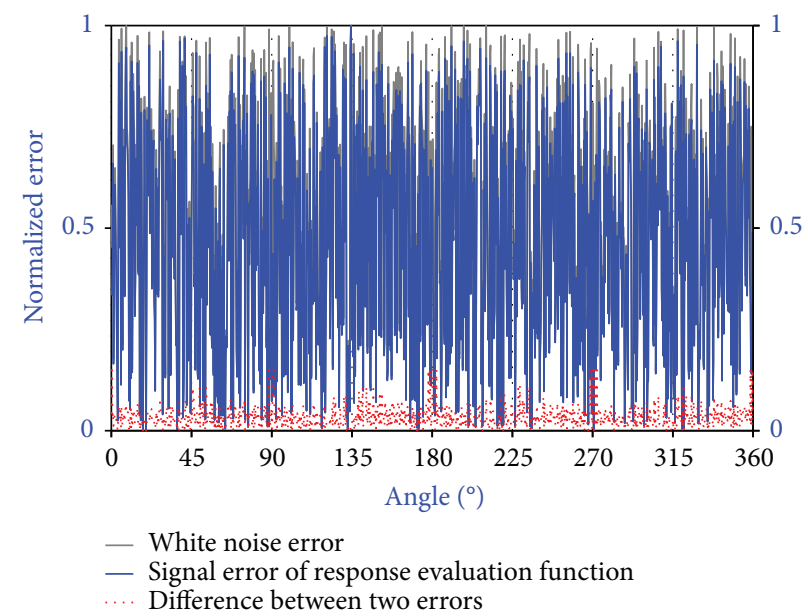

(c)

Figure 5: (a) Sine/cosine signal with white noise. (b) White noise error from quality evaluation function before modification. (c) White noise error from quality evaluation function after modification.

Step 4. Take (2) to conduct the response evaluation on the sensor.

Step 5. Modify the sensing response with (6) to find a minimized difference between the theoretical and optimal structure.

2.3. GA. Intelligent algorithms are employed for strategy facilitating and cost reducing $[17,18]$. Genetic algorithm, which is a class of optimization heuristics based on natural selection and genetic evolution, can be applied to problems where the variables to be optimized (genes) can be encoded to form a string (chromosome). Each string represents a trial solution of the problem. The GA operators exchange information between the strings to evolve new and better solutions [19]. Because the structure of magnetic-gratinglike stroke-sensing cylinder determines the working performance, this paper takes GA as the optimization model for variable design.

A multiparameter optimization problem using genetic algorithm is formulated by defining the system figure and the objective function of response quality, respectively. The modal parameters (i.e., natural modes) of the real structure are numerically obtained using its finite element model, from which the reduced numerical modes correspond to the target structure. The original related parameters are discussed in Section 3, where the characteristics of working elements are also described. The algorithm we use is implemented as follows:

(1) Initialization: consider a population of individuals, and each individual is described by one chromosome with $n$ genes, where each gene has $r$ alleles. Therefore, it has $r^{n}$ possible individuals [20]. Actual parameter information is contained within the components. The initial parameter values are randomly assigned.

(2) Fitness calculation: the optimum configuration is sought by minimizing the value of signal response quality. The fitness function of each individual in current population can be calculated according to (6). The fitness of total population is evaluated based on the outcome from the proposed function.

(3) Selection: the selection mechanism allows a small portion of chromosomes from the population for further evaluation [21]. Selection is accomplished by 
holding a fitness function among random competitors. The winner of the fitness function is the individual with the highest fitness, and the winner is then inserted into the mating pool [22]. In other words, chromosomes with higher fitness values are more likely selected to produce the next generation.

(4) Crossover: offspring is generated by two randomly selected parents exchanging genetic information with each other. The crossover step aims at leading to minor differences between parents and children, strengthening the exploitative power of reproduction [23].

(5) Mutation: mutation provides an opportunity to search new areas of the solution space. By randomly altering the alleles of genes, the GAs can effectively avoid trap situations and maintain sufficient variance in the population $[24,25]$, whereas the probability of mutation is around 1\%, since a high probability of mutation reduces the GA to a random search function.

(6) Replace the initial population with new individuals of best chromosome. An outcome value closer to the target value via repetitive iterative process can be generated. Consequently, after a large number of iterations, the best chromosome in the population is translated as the selected solution.

\section{Parameter Optimization}

A sensor's performance characteristics are governed by its specific architecture. The parameters with large uncertainty in the design process can affect the measurement accuracy significantly. Aiming at investigating the structure design for increasing working performance, optimization techniques are used to determine the design parameters. According to paragraph II, the key of measuring is the response of the sensor output. That is, in other words, given a parameterized representation of a sensor, we are capable of predicting its measurement accuracy.

To assist the optimization process, there are seven variables involved in this system (Table 1), which are diameter of permanent magnet $\left(S_{\mathrm{MD}}\right)$, height of permanent magnet $\left(S_{\mathrm{HM}}\right)$, distance from bottom of permanent magnet to centre of sensor $\left(S_{\mathrm{DM}}\right)$, distance from surface of piston rod to centre of sensor $\left(S_{\mathrm{DC}}\right)$, slot shape of piston rod $\left(f\left(y_{t}\right)\right)$, diameter of piston rod $\left(S_{\mathrm{CYD}}\right)$, and groove width of piston rod $\left(S_{\mathrm{PT}}\right)$. Based on the working principle, a novel magnetic-grating-like stroke-sensing cylinder is designed and its structural parameters are illustrated in Figure 6. The magnetic-grating-like stroke-sensing cylinder is for detecting the displacement of piston rod under working circumstance. Careful design of the structural variables can substantially improve measurement accuracy. Hence, the optimal structure design issue is formulated as an optimization problem. All the following parameters are transformed from the modeling and simulation methods into the function value of $Q_{T}$.
TABLE 1: Nomenclature.

\begin{tabular}{lc}
\hline Symbol & Definition \\
\hline$S_{\mathrm{MD}}$ & Diameter of permanent magnet \\
$S_{\mathrm{HM}}$ & Height of permanent magnet \\
$S_{\mathrm{DM}}$ & Distance from bottom of permanent magnet to \\
$S_{\mathrm{DC}}$ & centre of sensor \\
$f\left(y_{t}\right)$ & Distance from surface of piston rod to centre of sensor \\
$S_{\mathrm{CYD}}$ & Diameter of piston rod \\
$S_{\mathrm{PT}}$ & Groove width of piston rod \\
\hline
\end{tabular}

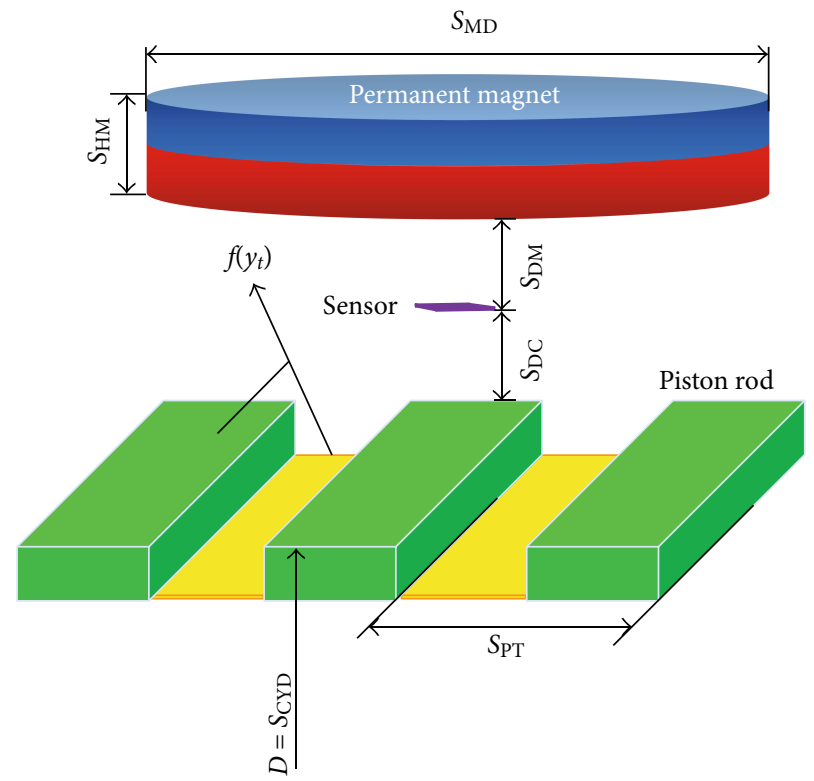

Figure 6: Parameter of magnetic-grating-like stroke sensing for optimization.

We notice that the diameter of piston rod in a suspended state, which avoids the direct contact between the magnet and the Hall sensor [26, 27], achieves the purpose of design without optimization. So $S_{\mathrm{CYD}}$ is set as defined value of $50 \mathrm{~mm}$. Due to the definition of measurement accuracy, the groove width of piston rod represents one working period for segmentation. Hence, the smaller the groove width is, the higher accuracy can be obtained. Since the highest cutting quality is $1 \mathrm{~mm}, S_{\mathrm{PT}}$ is given a balance value of $1.25 \mathrm{~mm}$ for machining.

The conceptual framework for optimizing a candidate design is shown in Figure 7. The optimal parameter value is searched using the signal response evaluation function, and the performance testing is deployed for model verification. The details of the signal response evaluation-based processes are described in what follows.

3.1. Effect of Distance from Surface of Piston Rod to Centre of Sensor. To validate every analytical result further, other parameters are assumed fixed during the optimizing process. The sensor exists in the position between the permanent magnet and the piston rod, and the distance is 


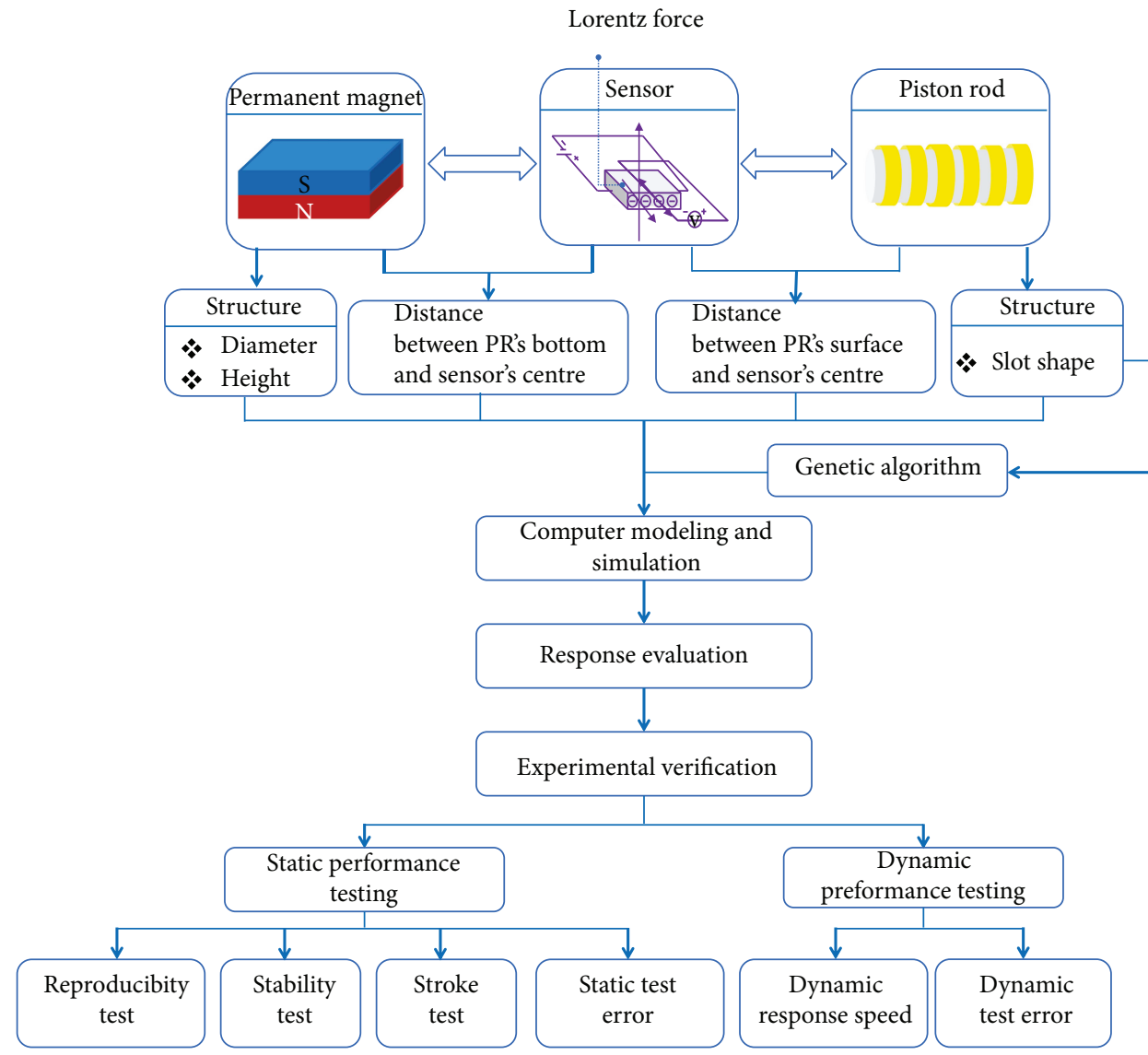

FIGURE 7: Conceptual diagram of magnetic-grating-like stroke-sensing cylinder for evolving structure model.

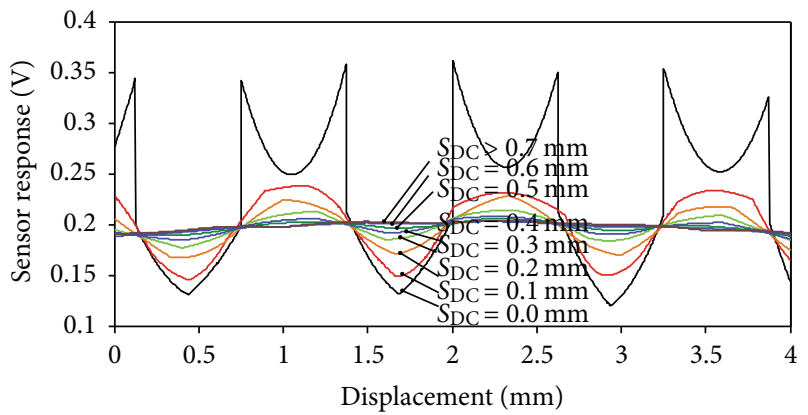

FIGURE 8: Signal response of $S_{\mathrm{DC}}$ on different displacements.

named as $S_{\mathrm{DC}}$. Typical parameters are quantified by selecting the proper value of ordinary used sensing systems, where $S_{\mathrm{MD}}=10 \mathrm{~mm}, S_{\mathrm{HM}}=10 \mathrm{~mm}, S_{\mathrm{DM}}+S_{\mathrm{DC}}=8 \mathrm{~mm}, S_{\mathrm{PT}}=1.25$ $\mathrm{mm}, S_{\mathrm{CYD}}=50 \mathrm{~mm}$ and $f\left(y_{t}\right)$ is the shape of equally spaced rectangular, which is further optimized for showing that how these values are suitable for design purposes. The variation of $S_{\mathrm{DC}}$ is typically taken from 0 to $5 \mathrm{~mm}$ with the step of $0.1 \mathrm{~mm}$. Figure 8 shows the response curve of the Hall sensor with different values of $S_{\mathrm{DC}}$ by simulation.

It can be seen that the signal intensity is affected by the distance between the Hall sensor and the piston rod. As seen here, a smaller distance has a stronger sensor response while the value over $0.7 \mathrm{~mm}$ changes only slightly the strength of sensor response. However, $S_{\mathrm{DC}}=0$ results in the severe signal

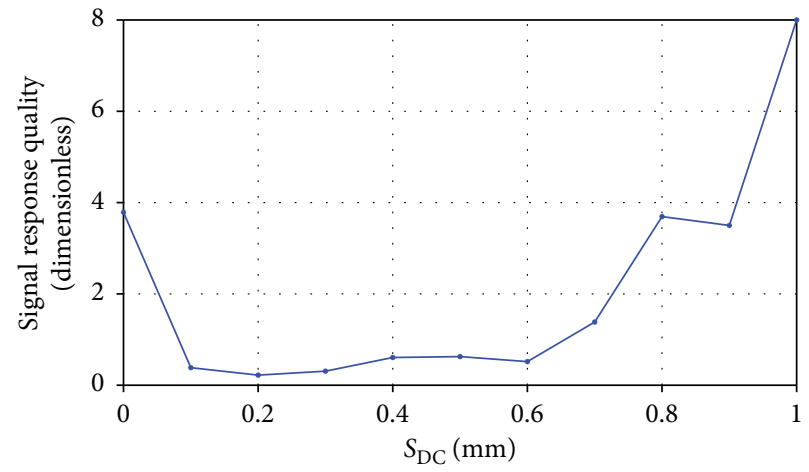

FIgURE 9: Effect of different $S_{\mathrm{DC}}$ on response quality.

distortion. To evaluate the quality of signal response, Figure 9 shows the derivation results depending on the variation of $S_{\mathrm{DC}}$ with Equation (6) applied. The parameter value from $0.1 \mathrm{~mm}$ to $0.6 \mathrm{~mm}$ is shown to be in the desired position.

3.2. Effect of Diameter of Permanent Magnet. The variation of permanent magnet shape has a direct effect on the magnetic induction, which is involved with the diameter $S_{\mathrm{MD}}$ and the height $S_{\mathrm{HM}}$. Thereby, both of them can be analyzed by this method. We take $S_{\mathrm{DC}}=0.3 \mathrm{~mm}$ according to the aforementioned outcome while other parameters are set as before. The signal response of variable parameter $S_{\mathrm{MD}}$ is shown in 


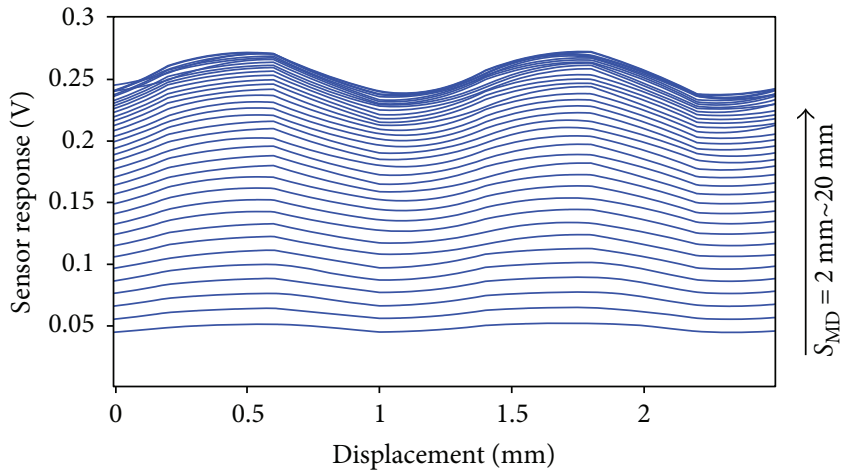

FIGURE 10: Signal response of different values of $S_{\mathrm{MD}}$ on different displacements.

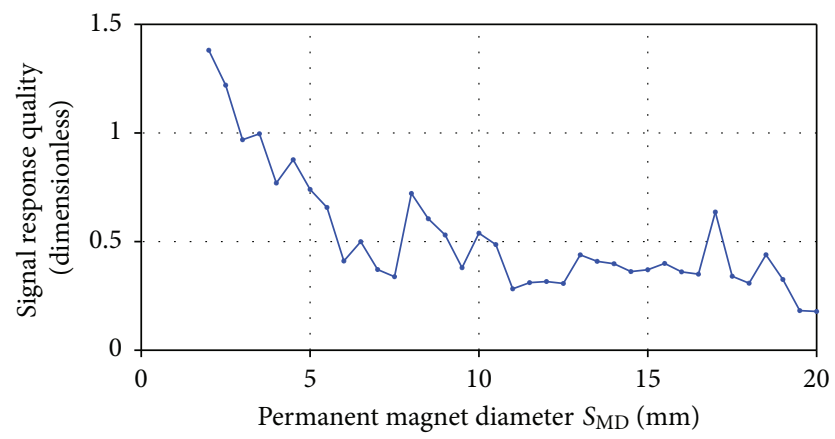

FIGURE 11: Effect of different $S_{\mathrm{MD}}$ on response quality.

Figure 10. At the beginning, the sensor response is small when $S_{\mathrm{MD}}$ is small. The sensing signal response increases with the increasing of diameter and reaches a maximum value when the parameter is in the range $16-20 \mathrm{~mm}$, but the maximum value is not influenced very much.

The signal response quality is found to be improved and stabilized as diameter increases to over $10 \mathrm{~mm}$ as shown in Figure 11. Considering the sensing signal strength outcome, the optimal value $S_{\mathrm{MD}}=16 \mathrm{~mm}$ is taken.

3.3. Effect of Height of Permanent Magnet. For the height of permanent magnet $S_{\mathrm{HM}}$, a similar analysis result is yielded as shown in Figure 12. In the range of $0-30 \mathrm{~mm}$, the output of Hall sensor increases while the difference in the response strength between $30 \mathrm{~mm}$ and $40 \mathrm{~mm}$ is not distinguished.

Figure 13 depicts the relation between signal response quality and the height of permanent magnet. The quality of sensing response fluctuates significantly in the range of $0-5 \mathrm{~mm}$ while slightly in $5 \mathrm{~mm}-35 \mathrm{~mm}$. Particularly, it decreases slightly when $S_{\mathrm{HM}} \geq 35 \mathrm{~mm}$. In line with actual requirement of high accuracy, the optimal $S_{\mathrm{HM}}$ set at the value of $35 \mathrm{~mm}$ can be suggested.

\subsection{Effect of Distance from Bottom of Permanent Magnet to} Surface of Piston Rod. The mechanical position of permanent magnet plays an important role in changing the sensing signal as well. The physical distance between the permanent magnet and the piston rod $S_{\mathrm{DM}}+S_{\mathrm{DC}}$ is taken as typically designed parameter. For the typically selected parameters

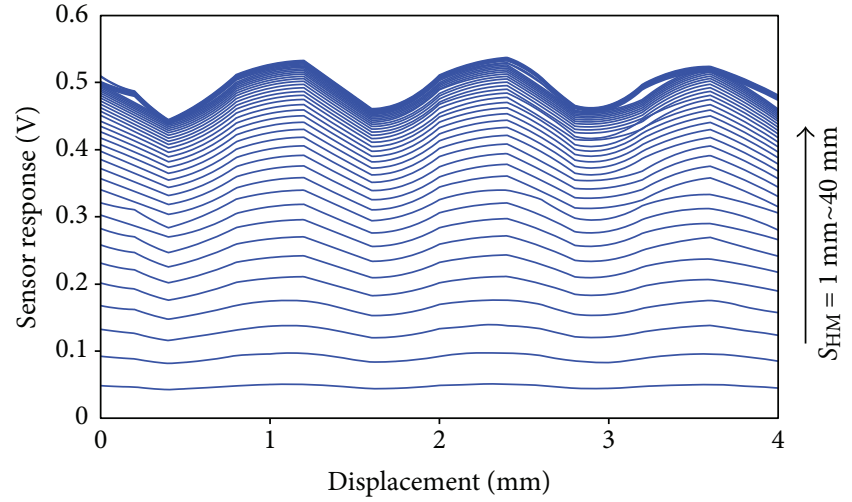

Figure 12: Signal response of $S_{\mathrm{HM}}$ on different displacements.

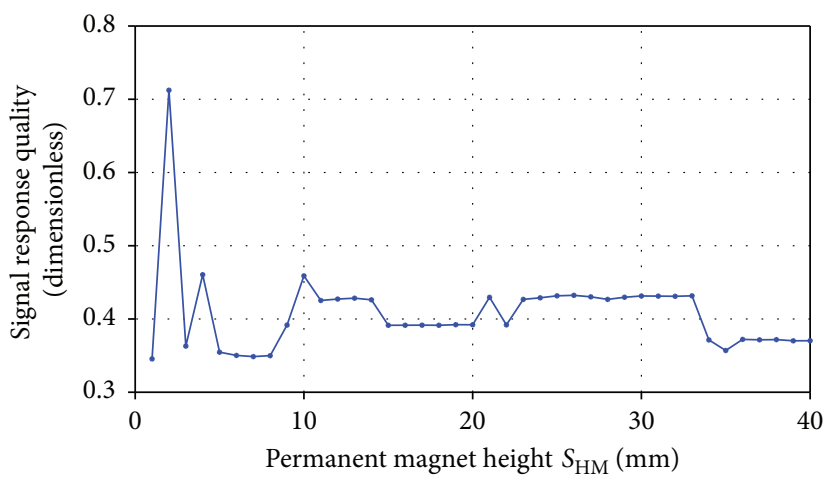

FIGURE 13: Effect of different $S_{\mathrm{HM}}$ on response quality.

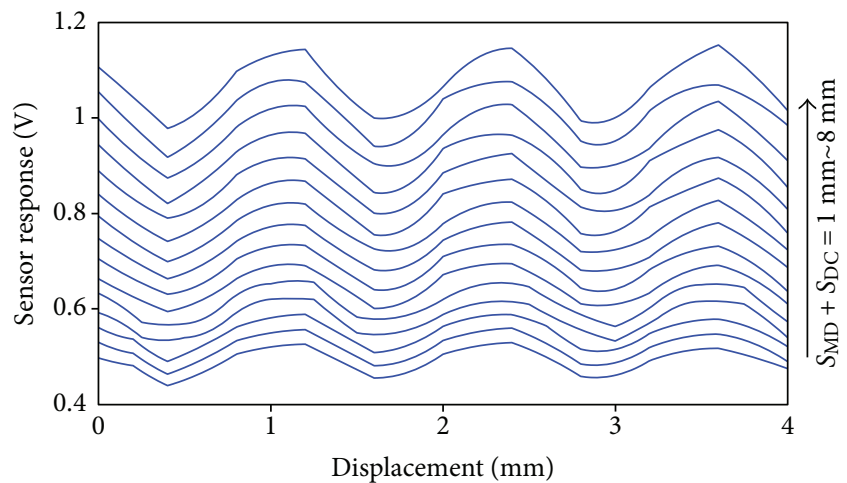

FIGURE 14: Signal response of $S_{\mathrm{DM}}+S_{\mathrm{DC}}$ on different displacements.

optimized above, the effect of $S_{\mathrm{DM}}+S_{\mathrm{DC}}$ on performance, with variation step of $0.5 \mathrm{~mm}$, can be observed in Figure 14 .

As seen here, the decreasing distance causes the increasing of signal response. However, the computing results of parameter values illustrate that the characteristic of piston rod can be clearly described at all values. In the practical design, different structures correspond to different locations of permanent magnet. The objective function curve of signal response quality is shown in Figure 15. When the distance increases $(\geq 5.5 \mathrm{~mm})$, the function value changes much greater than that of short distance, which indicates that a small value of $S_{\mathrm{DM}}+S_{\mathrm{DC}}$ is more appropriate for practical use. The optimal $S_{\mathrm{DM}}+S_{\mathrm{DC}}$ is given as $5 \mathrm{~mm}$. 


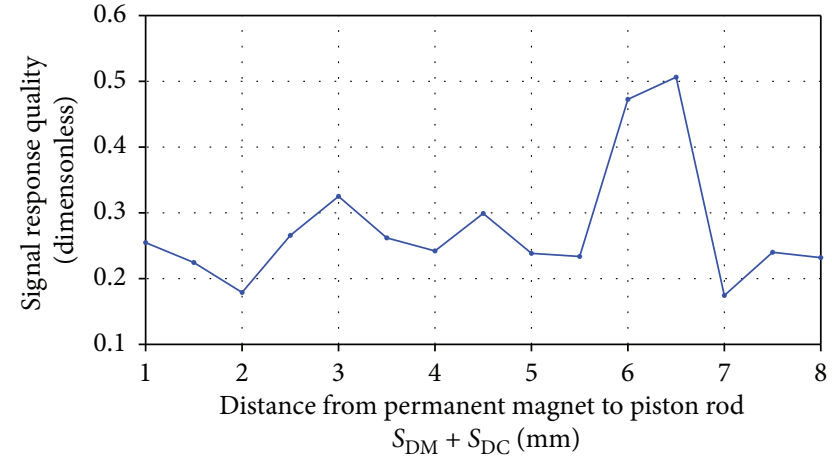

FIGURE 15: Effect of different $S_{\mathrm{DM}}+S_{\mathrm{DC}}$ on response quality.

3.5. Effect of Slot Shape of Piston Rod. The shape of piston rod slot is optimized by setting up the coordinate system of $f\left(y_{t}\right)$ in Figure 16. The slot is described in plane $X$. Axis $Y$ is the axle centre of piston rod, and axis $Z$ is the start of periodic variation in slot shape. For a specific architecture, however, it is possible to define the best slot which is defined with the optimum "set" of the geometrical parameters [28]. Therefore, nine anchor points $\left(V_{0}, V_{1}, \ldots, V_{8}\right)$ from $A_{1}$ to $B_{1}$ are picked up for analysis. For simplicity, the anchor points are evenly distributed between the dot and $Y=S_{\mathrm{PT}} / 2$. Considering the machining process, the values from $A_{1}$ to $B_{1}$ decrease monotonically. The optimization problem is formulated based on the design of these nine parameters. Constraints in (7) are then formulated to reject infeasible slot geometries.

$$
\begin{aligned}
& V_{0}=\left(0, y_{0}, z_{0}\right), \\
& V_{1}=\left(0, y_{0}+S_{\mathrm{PT}} \cdot \frac{1}{16}, z_{0}-\Delta Z_{1}\right), \\
& V_{2}=\left(0, y_{0}+S_{\mathrm{PT}} \cdot \frac{2}{16}, z_{0}-\Delta Z_{2}\right), \\
& V_{3}=\left(0, y_{0}+S_{\mathrm{PT}} \cdot \frac{3}{16}, z_{0}-\Delta Z_{3}\right), \\
& V_{4}=\left(0, y_{0}+S_{\mathrm{PT}} \cdot \frac{4}{16}, z_{0}-\Delta Z_{4}\right), \\
& V_{5}=\left(0, y_{0}+S_{\mathrm{PT}} \cdot \frac{5}{16}, z_{0}-\Delta Z_{5}\right), \\
& V_{6}=\left(0, y_{0}+S_{\mathrm{PT}} \cdot \frac{6}{16}, z_{0}-\Delta Z_{6}\right), \\
& V_{7}=\left(0, y_{0}+S_{\mathrm{PT}} \cdot \frac{7}{16}, z_{0}-\Delta Z_{7}\right), \\
& V_{8}=\left(0, y_{0}+S_{\mathrm{PT}} \cdot \frac{8}{16}, z_{0}-S_{\mathrm{PH}}\right),
\end{aligned}
$$

where $y_{0}=0, z_{0}=S_{\mathrm{CYD}} / 2$, and $\Delta Z_{i}(i=1,2, \ldots, 7)$ is the variable quantity of $Z$-direction. Initially, the relationship of $\Delta Z_{i}$ can also be written as

$$
0 \leq \Delta Z_{1} \leq \Delta Z_{2} \leq \Delta Z_{3} \leq \Delta Z_{4} \leq \Delta Z_{5} \leq \Delta Z_{6} \leq \Delta Z_{7} \leq S_{\mathrm{PH}},
$$

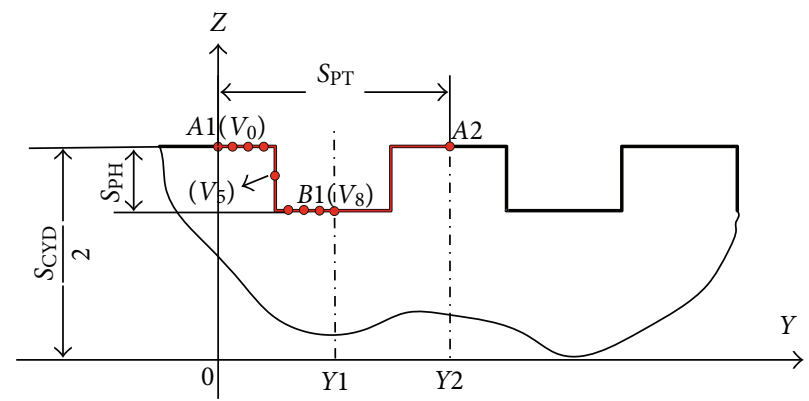

Figure 16: Geometric illustration of the slot.

whereas the variation of these parameters is not constrained but depends on the optimization outcome. Since the values of $S_{\mathrm{CYD}}, S_{\mathrm{PT}}$, and $S_{\mathrm{PH}}$ are defined earlier, the sensor response determined by optimal parameter set can be expressed as

$$
f_{\text {so }}(t)=f\left(t, S_{\mathrm{DC}}, \Delta Z_{1}, \Delta Z_{2}, \Delta Z_{3}, \Delta Z_{4}, \Delta Z_{5}, \Delta Z_{6}, \Delta Z_{7}\right),
$$

where $f_{\text {so }}$ is the response and $t$ is the specific working moment of Hall element. Aiming at acquiring a harmonic signal, the response evaluation function $Q_{T}$ is introduced as the objective function.

$$
Q_{T}=\operatorname{Max}\left\{\frac{\left|f_{\mathrm{tct}}\left(\theta_{T_{-} i}\right)-f_{\mathrm{tct}}\left(\theta_{s_{-} i}\right)\right| \times 0.017455}{f_{\mathrm{Ad}}\left(\theta_{s_{-} i}\right)}\right\} .
$$

In the relationship, $f_{\text {tct }}\left(\theta_{T_{-} i}\right)$ stands for the tangent/ cotangent of response incorporated which can be derived as

$$
\begin{aligned}
& f_{\mathrm{tct}}\left(\theta_{T_{-} i}\right) \\
& = \begin{cases}\left|\frac{f_{\mathrm{so}}\left(T_{-} i\right)}{f_{\mathrm{so}}\left(T_{-} i+1 / 4 T\right)}\right|, & \left|f_{\mathrm{so}}\left(T_{-} i\right)\right| \leq\left|f_{\mathrm{so}}\left(T_{-} i+1 / 4 T\right)\right|, \\
\left|\frac{f_{\mathrm{so}}\left(T_{-} i+1 / 4 T\right)}{f_{\mathrm{so}}\left(T_{-} i\right)}\right|, & \left|f_{\mathrm{so}}\left(T_{-} i\right)\right|>\left|f_{\mathrm{so}}\left(T_{-} i+1 / 4 T\right)\right|,\end{cases}
\end{aligned}
$$

where $T$ is the signal outcome period. Thus, the shape optimization of the slot is transferred into detecting the minimum value of $Q_{T}$.

A GA is employed to search the optimum solution of $Q_{T}$. A flowchart that describes the GA optimization procedure that has been adapted in this paper is shown in Figure 17. The input to GA is $Q_{T}$, and the output is the geometry of the slot.

The process starts with randomly generated population. Each chromosome represents a possible set of parameters associated with a fitness value. The total population of each generation is evaluated based on slot geometry where GA carries out a fitness-based selection. In our GA, the size of the population is chosen to be 40 on the basic of modeling and modification. Processes are subject to recombination to form a successor population. The single-point crossover is used, and the probability of mutation is set as $0.17 \%$. During recombination, chromosomes with the higher fitness values 


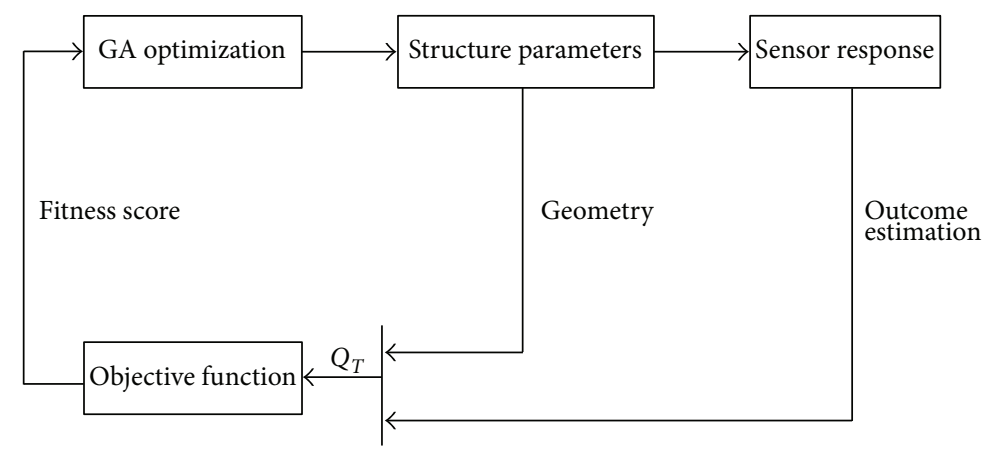

FIgURE 17: GA optimization procedure.

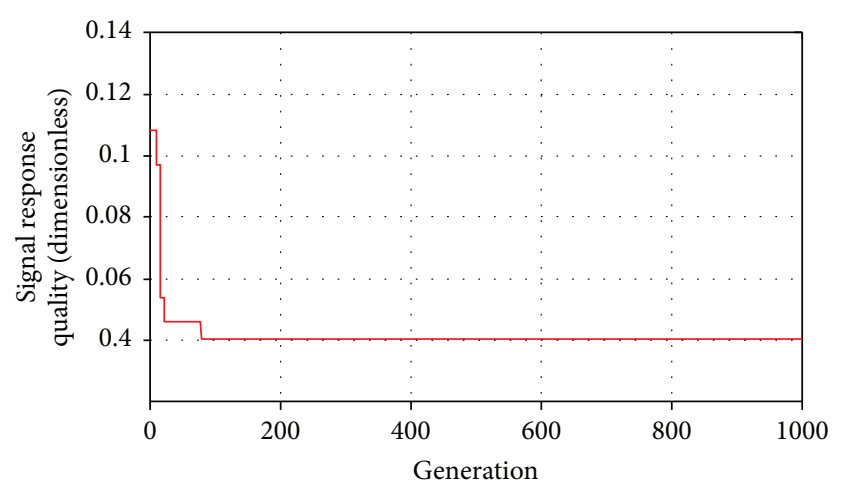

FIGURE 18: Evolution process of best (smallest) quality evaluation function.

are more likely to be selected from the population, which passed on to the successor population. This is an iterative process, through which various generations are evolved until some stopping criterion is specified, and an optimal solution is reached based on an increase. The GA search process for the quality evaluation function is shown in Figure 18. At the beginning of evolution process (about less than 40 generations), the differences between every individual are relatively large, which results in individuals with low fitness being eliminated and ones with higher fitness being saved. After certain generations (about larger than 100 generations), the variation in target value is small, finally approaching to a constant value of 0.04 .

Accordingly, a piston rod with optimized variables is obtained. Configuration uses the following parameter values: $Q_{T}=0.0520, S_{\mathrm{DC}}=0.4, \Delta Z_{1}=0.0146, \Delta Z_{2}=0.0440, \Delta Z_{3}=$ $0.0633, \Delta Z_{4}=0.0781, \Delta Z_{5}=0.0749, \Delta Z_{6}=0.0665, \Delta Z_{7}=$ 0.0427 . Figure 19 shows the photograph of the manufactured piston rod as well as its actual signal response with optimal pattern. Some signal fluctuation appears randomly within the sine signal response period due to the machining error, which can be eliminated by improving the accuracy of machining technology.

\section{Experiment and Analysis}

4.1. Experimental System. The experiments are conducted to verify the working performance of magnetic-grating-like stroke-sensing cylinder. The hardware used to perform the experiments is shown in Figure 20. Together, the linear slide and the fixed bearing house the piston rod and the permanent magnet, respectively, on the supporting base. A high-accuracy grating is placed parallel with the piston rod for precisely detecting the displacement. The sensor output is recorded with the comparison of the grating. During the test, the permanent magnetic is fixed while the piston rod is in reciprocating linear motion. As explained in Section 1, the displacement is then calculated from sensing signal of Hall sensor. Experiments are carried out in an air-conditioned room maintained almost at constant temperature, humidity, and pressure. According to the JJG 6442003 "Verification Regulation of Vibration Displacement Transducer," much work is devoted to assess the static and dynamic performance.

\subsection{Static Testing Configuration}

4.2.1. Stroke Test. Stroke tests contain short-term test and long-term test. Both are basically designed for testing the error between theoretical stroke and experimental stroke. The working distance of short-term test is one grating, which is one thread lead of the piston rod. The output of Hall sensor is detected every $5^{\circ}$, and the subdivision points is 96 . Figure 21 shows the results of short-term test, where $S_{\mathrm{REL}}$ is the sampled data point and $S_{\mathrm{TT}}$ is the actual one. The maximum drift of short-term test is $\Delta_{\mathrm{st}}=0.031 \mathrm{~mm}$.

Similarly, the long-term test takes 100 continuous grating as the working distance. The maximum error of long-term is $\Delta_{\mathrm{lt}}=0.037 \mathrm{~mm}$. The distribution of all the recorded points is presented in Figure 22, which shows the range of output error in forward and backward stroke. The numerical difference is $0.045 \mathrm{~mm}$ in maximum.

4.2.2. Stability Test. The magnetic flux density is addressed relying on highly stabilized system output. In the power supply driving mode, the sensor is of different position of the stroke. The pass/fail of stability is based on the output of the sensor. The voltage is kept at $24.0 \mathrm{~V}$ for 1 hour by use of highly stabilized power supply, and the displacement is measured every 2 hours. The corresponding displacements $S_{\mathrm{RV}}$, obtained after sensing steps, are given in Table 2 .

Arithmetic average difference can be calculated during relevant periods. The value of magnetic flux density stability error 


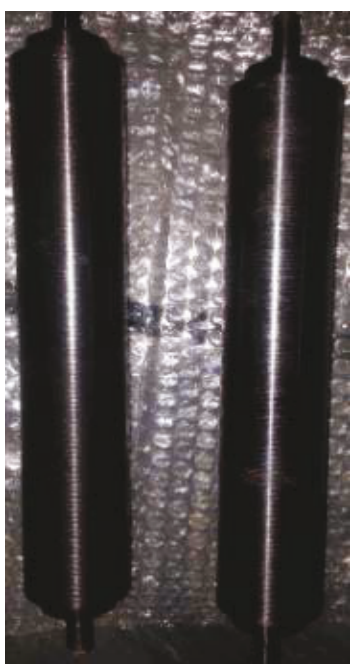

(a)

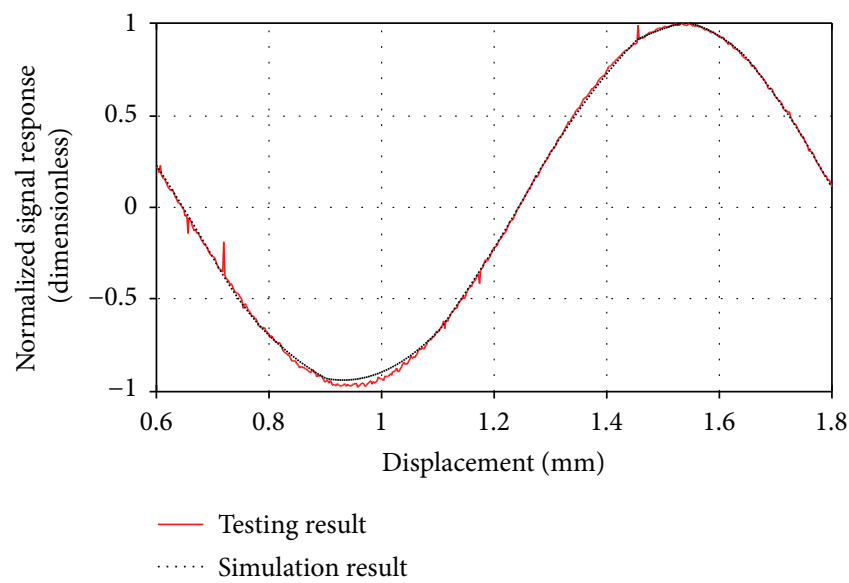

(b)

Figure 19: Photograph of piston rod sample and its signal response.

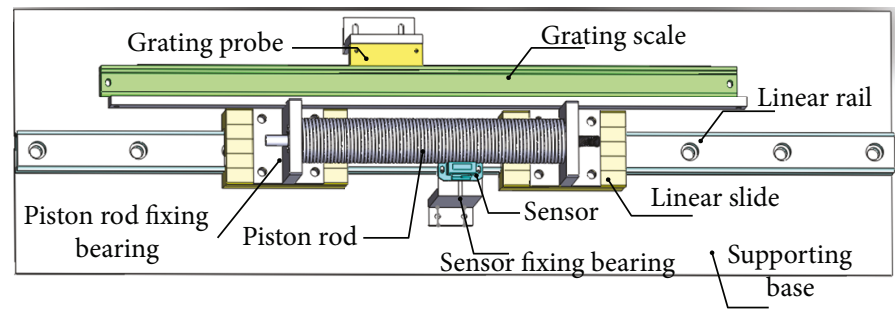

Figure 20: Schematic layout of test setup.

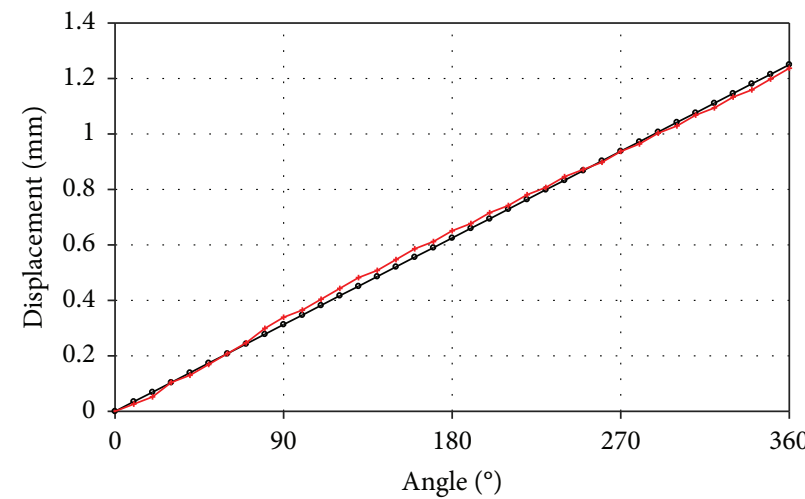

$\rightarrow S_{\mathrm{REL}}$

$\longrightarrow$ Forward stroke $S_{\mathrm{TT}}$

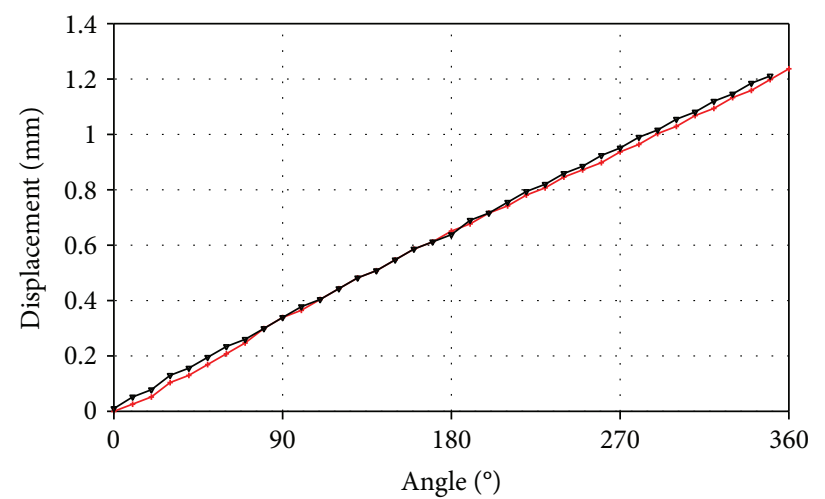

- Forward stroke $S_{\text {TT }}$

$\rightarrow$ Backward stroke $S_{\mathrm{TT}}$

FIgURE 21: Output of theoretical and actual stroke.

$$
\Delta_{\mathrm{rv}}=\frac{\left[\max \left(S_{\mathrm{RV} 1}\right)-\min \left(S_{\mathrm{RV} 1}\right)\right]+\left[\max \left(S_{\mathrm{RV} 2}\right)-\min \left(S_{\mathrm{RV} 2}\right)\right]}{2}
$$

is $0.0195 \mathrm{~mm}$ in the power supply driving mode.

4.2.3. Reproducibility Test. The reproducibility test is designed to identify the repetitive feature of sensor output within the same stroke. The calibration is conducted three times while the piston rod repeats in a defined path. The recorded values, for each measurement point, are shown in Table 3 and indicated, at each point, the maximum difference within all test results characterizing the reproducibility error $\Delta_{\text {ri }}$ which can be written as

$$
\begin{gathered}
\Delta_{\mathrm{ri}}=\max \left\{\max \left|S_{\mathrm{RPV} 1}-S_{\mathrm{RPV} 2}\right|, \max \left|S_{\mathrm{RPV} 1}-S_{\mathrm{RPV} 3}\right|,\right. \\
\left.\max \left|S_{\mathrm{RPV} 3}-S_{\mathrm{RPV} 2}\right|\right\}=0.026 \mathrm{~mm},
\end{gathered}
$$

where $S_{\mathrm{RPV} 1}, S_{\mathrm{RPV} 2}$, and $S_{\mathrm{RPV} 3}$ represent the measurement data set of these calibration tests. 


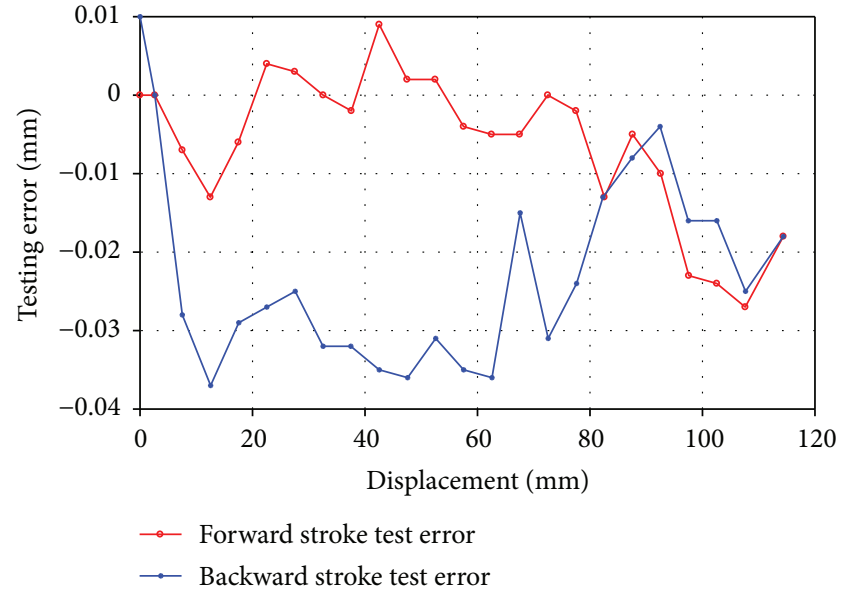

Figure 22: Test error of forward and backward stroke.

TABLE 2

(a) Sensor output of stability test 1

\begin{tabular}{lc}
\hline Time $(\mathrm{h})$ & $S_{\mathrm{RV} 1}(\mathrm{~mm})$ \\
\hline 0 & 62.539 \\
2 & 62.526 \\
4 & 62.539 \\
6 & 62.526 \\
8 & 62.526 \\
12 & 62.539 \\
14 & 62.539 \\
16 & 62.526 \\
18 & 62.539 \\
20 & 62.526 \\
22 & 62.539 \\
24 & 62.539 \\
\hline
\end{tabular}

(b) Sensor output of stability test 2

\begin{tabular}{lc}
\hline Time $(\mathrm{h})$ & $S_{\mathrm{RV} 2}(\mathrm{~mm})$ \\
\hline 0 & 107.565 \\
2 & 107.552 \\
4 & 107.565 \\
6 & 107.578 \\
8 & 107.578 \\
12 & 107.552 \\
14 & 107.565 \\
16 & 107.552 \\
18 & 107.578 \\
20 & 107.565 \\
22 & 107.565 \\
24 & 107.578 \\
\hline
\end{tabular}

4.2.4. Static Error. The absolute static error $\Delta_{\text {se }}$ expresses the difference between the real and ideal characteristics, which has the form as follows.
TABLE 3: Sensor output of reproducibility test 1.

\begin{tabular}{lcc}
\hline$S_{\mathrm{RPV} 1}(\mathrm{~mm})$ & $S_{\mathrm{RPV} 2}(\mathrm{~mm})$ & $S_{\mathrm{RPV} 3}(\mathrm{~mm})$ \\
\hline 0.000 & 0.000 & 0.000 \\
2.995 & 2.995 & 2.982 \\
6.042 & 6.029 & 6.029 \\
9.010 & 8.984 & 9.010 \\
12.005 & 12.005 & 12.031 \\
15.039 & 15.026 & 15.039 \\
17.969 & 17.982 & 17.995 \\
21.016 & 21.029 & 21.029 \\
24.010 & 23.997 & 23.997 \\
27.005 & 26.992 & 27.005 \\
30.000 & 30.000 & 30.013 \\
32.982 & 32.956 & 32.956 \\
36.003 & 36.003 & 36.029 \\
39.010 & 38.984 & 38.997 \\
41.979 & 41.966 & 41.979 \\
45.013 & 45.013 & 45.026 \\
47.969 & 47.969 & 47.982 \\
51.003 & 51.003 & 51.029 \\
53.997 & 53.984 & 54.010 \\
56.992 & 56.979 & 56.992 \\
60.039 & 60.039 & 60.039 \\
62.982 & 62.969 & 62.982 \\
66.003 & 66.003 & 69.029 \\
69.023 & 69.010 & \\
\hline & & \\
& & \\
& &
\end{tabular}

where $\Delta_{\mathrm{st}}, \Delta_{\mathrm{lt}}, \Delta_{\mathrm{rv}}, \Delta_{\mathrm{ri}}$, and $\Delta_{\mathrm{m}}$ are errors caused by shortterm variations, long-term variations, sensing instability, data agreement uncertainty, and variance of machining accuracy, respectively. The actual length of piston $\operatorname{rod}$ is $l=300$ $\mathrm{mm}$, and the parallelism error is $d=0.02 \mathrm{~mm}$ according to machining technology. Hence, the measurement error in relationship with mechanical accuracy is

$$
\Delta_{\mathrm{m}}=\frac{l}{\cos [a \tan (d / l)]}-l \text {. }
$$

The error determined by machining deviation is $7 \times$ $10^{-7} \mathrm{~mm}$, which is however an insignificant contributing factor. Thus, the static error of magnetic-grating-like strokesensing cylinder is $\Delta_{\mathrm{se}}=0.0492 \mathrm{~mm}$.

\subsection{Dynamic Testing Configuration}

4.3.1. Dynamic Error. In this research, the dynamic test deals with the working accuracy of different measuring modes. The setup for evaluating the dynamic error is as follows: a lowspeed $(0.1 \mathrm{~m} / \mathrm{s})$ and a high-speed $(0.3 \mathrm{~m} / \mathrm{s})$ measurement stroke are carried out three times with the sampling rate of 100 Ksps. 


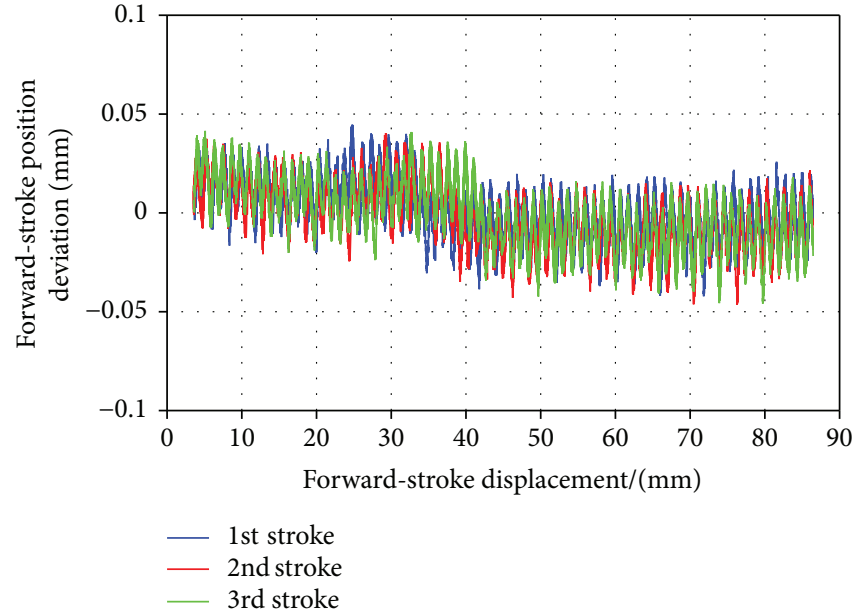

(a)

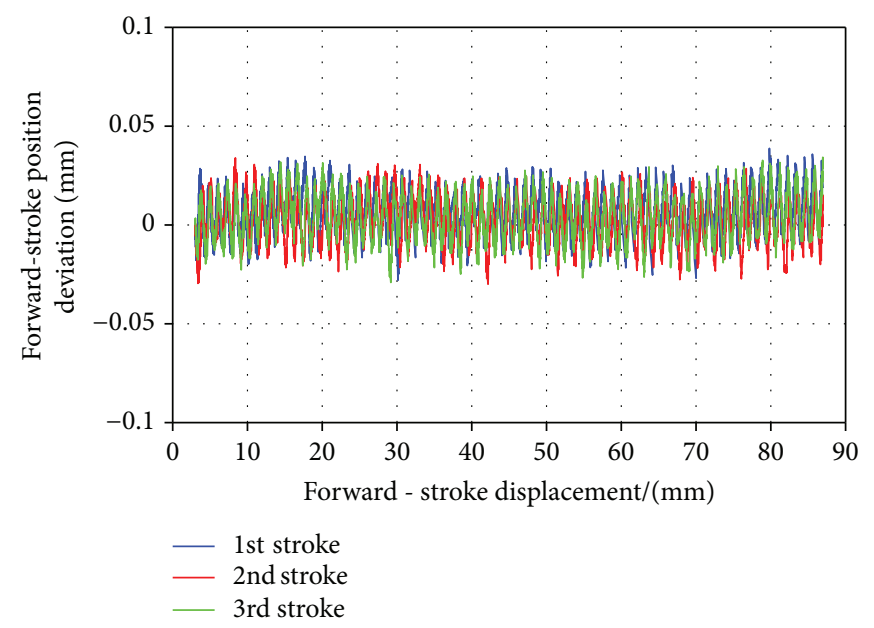

(c)

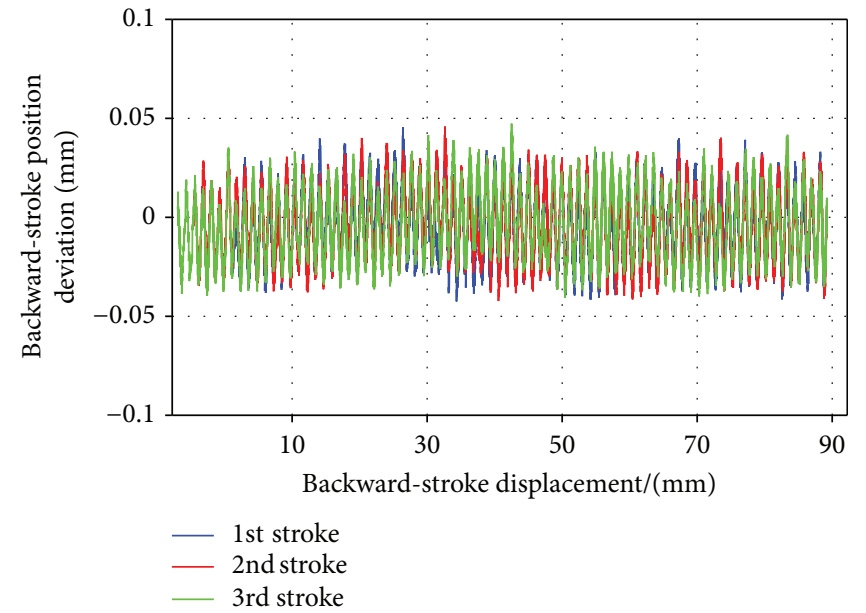

(b)

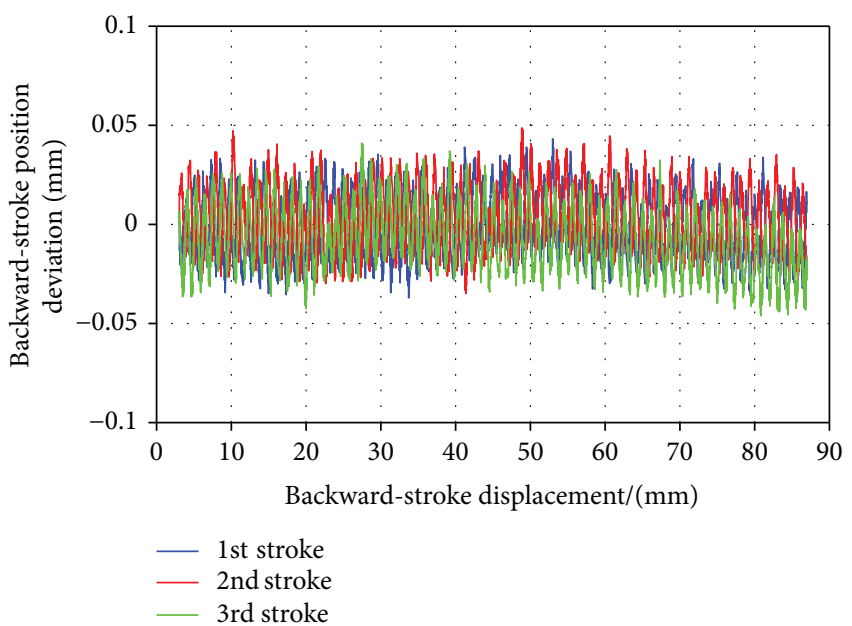

(d)

FIGURE 23: (a) Forward-stroke output in low speed. (b) Backward-stroke output in low speed. (c) Forward-stroke output in high speed. (d) Backward-stroke output in high speed.

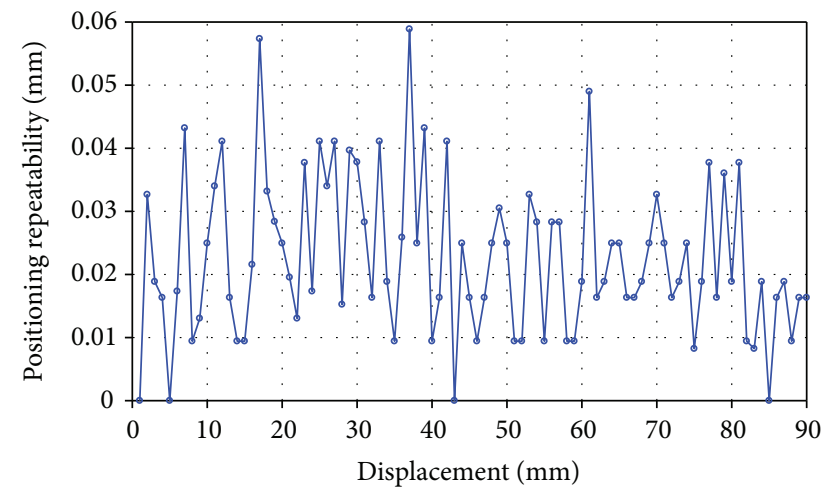

FIGURE 24: Positioning accuracy of dynamic testing.

Dynamic error is evaluated in accordance with the definition in [29]. The target position is $P_{i}$ where $i$ identifies the particular position among other selected target positions along the stroke. $P_{i j}$ is the measured position reached by the functional point on the $j$ th approach to the $i$ th target position. Then, the positioning deviation is

$$
X_{i j}=P_{i j}-P_{i}
$$

and the mean positioning deviation is

$$
\overline{X_{i}}=\frac{1}{n} \sum_{j=1}^{n} X_{i j} .
$$

The standard uncertainty of the positioning deviations obtained by a series of $n$ approaches at a position $P_{i}$ is

$$
S_{i}=\left[\frac{1}{n-1} \sum_{j=1}^{n}\left(X_{i j}-\overline{X_{i}}\right)^{2}\right]^{1 / 2} .
$$

Taking a coverage factor $k=2$, the positioning repeatability at a position $P_{i}$ is 


$$
R_{i}=4 S_{i}
$$

We take the maximum value of the positioning repeatability at any position $P_{i}$ as the sensor repeatability:

$$
R=\max \left[R_{i}\right] .
$$

The difference between the algebraic maximum and minimum of the positioning deviations at any position $P_{i}$ is the systematic positioning error $\mathrm{El}$, which can be written as

$$
\mathrm{El}=\max \left[X_{i}\right]-\min \left[X_{i}\right]
$$

Now, the coverage factor $k=2$, the sensor positioning accuracy derived from the combination of systematic positioning errors and the estimator for positioning repeatability, is

$$
A_{S}=\max \left[X_{i}+2 S_{I}\right]-\min \left[X_{i}-2 S_{i}\right] .
$$

The standard deviation of measured values is

$$
\sigma=\left[\frac{1}{n} \sum_{i=1}^{n}\left(S_{i}-\overline{S_{i}}\right)^{2}\right]^{1 / 2} .
$$

In the working displacement of $0-90 \mathrm{~mm}$, recordings corresponding to speed and position variation are shown in Figure 23.

The position deviation of proposed sensing stroke is within the range of $\pm 0.05 \mathrm{~mm}$, and the error is found to be stable and repeatable. Based on the equations above, the positioning accuracy of different working distances is deduced (Figure 24).

As shown in Figure 24, different position tracking results in the variation of positioning accuracy. The working errors at any point involved with stroke sensor are $R= \pm 0.03 \mathrm{~mm}$ and $\mathrm{El}=0.0350 \mathrm{~mm}$. It can also be obtained that the sensor positioning accuracy is $A_{\mathrm{S}}= \pm 0.04695 \mathrm{~mm}$ and standard deviation of measured values is $\sigma=0.0031 \mathrm{~mm}$.

\section{Conclusion}

In this paper, the devising and deploying of magneticgrating-like stroke-sensing cylinder are specifically studied, according to its working mechanism and distinctive structure. Motivated by the significance of developing a highaccuracy displacement sensing device, capable of operating in different working distances and optimizing its performance, we set out to define the signal response quality of working parameters. The signal response quality evaluation algorithm is constructed to characterize sensor response in developing a stroke-sensing cylinder, which leads to the evaluation of structure variables mathematically. The optimal displacement sensor combines high response and state-ofthe-art signal segmentation method.

The prototype is processed and tested. The proposed testing workbench, together with the magnetic-gratinglike stroke-sensing cylinder, is set up in laboratory. Based on the static and dynamic testing, experimental outputs are recorded and demonstrate that it has a stable, repeatable performance and high measurement accuracy for a wide range of stroke. The testing results characterize the magnetic-grating-like stroke-sensing cylinder, which can replace current measurement devices and fulfill the demand of high-accuracy displacement detection.

\section{Conflicts of Interest}

The authors declare that they have no conflicts of interest.

\section{Acknowledgments}

This research is a general project supported by Shanxi Province Science Foundation for Youths (2015021123) and Shanxi Provincial Key Laboratory Open Fund (XJZZ201605).

\section{References}

[1] C. Bauer, K. Lagadec, C. Bès, and M. Mongeau, "Flight control system architecture optimization for fly-by-wire airliners," Journal of Guidance, Control, and Dynamics, vol. 30, no. 4, pp. 1023-1029, 2007.

[2] H. T. QI, Y. L. Fu, X. Y. Qi, and Y. Lang, “Architecture optimization of more electric aircraft actuation system," Chinese Journal of Aeronautics, vol. 24, no. 4, pp. 506-513, 2011.

[3] P. M. Churn, C. J. Maxwell, N. Schofield, D. Howe, and D. J. Powell, "Electro-hydraulic actuation of primary flight control surfaces," in IEE Colloquium on All Electric Aircraft (Digest No. 1998/260), vol. 15, pp. 3/1-3/5, London, UK, 1999.

[4] Z. Y. Xue, Study on the Power Support Dynamic Test and Control System, [Ph.D. thesis], Taiyuan University of Technology, China, 2003.

[5] X. N. Zhang, Y. Wang, and Y. L. Fu, "Symmetric control of asymmetric cylinder," Journal of Beijing University of Aeronautics and Astronautics, vol. 33, no. 11, pp. 1334-1339, 2007.

[6] T. Murakami and M. Kitsunai, "A hydraulic cylinder with a magnetic stroke sensor," European Journal of Pharmacology, vol. 347, no. 2, pp. 329-335, 1992.

[7] M. C. Lee, M. H. Lee, Y. J. Cho, S. Y. Yang, and K. S. Yoon, “On development of stroke sensing cylinder for automatic excavator," in 1995 Proceedings of the IEEE International Symposium on Industrial Electronics, pp. 363-368, Athens, Greece, June 1995.

[8] S. Y. Yang, M. C. Lee, M. H. Lee, and S. Arimoto, "Measuring system for development of stroke-sensing cylinder for automatic excavator," IEEE Transactions on Industrial Electronics, vol. 45, no. 3, pp. 376-384, 1998.

[9] S. Y. Yang, M. C. Lee, M. H. Lee, and S. Arimoto, "Development of digital stroke sensing cylinder and its performance evaluation," Robotica, vol. 14, no. 06, pp. 687-694, 1996.

[10] B. Liu and J. Y. Li, "Research on signal subdivision of grating sensor," in Proceedings of 2011 6th International Forum on Strategic Technology, pp. 1235-1238, Harbin, Heilongjiang, Aug. 2011.

[11] R. Guo, Research on Moiré Fringe Signal Subdivision Technology Based on Incremental Optical Encoder [Ph.D. thesis], Nanjing University of Aeronautics and Astronautics, China, 2013.

[12] Y. J. Li, Research on Grating Signal Subdivision Based on Amplitude Sampling Technology [Ph.D. thesis], Harbin University of Science and Technology, China, 2013. 
[13] X. J. Lin, Y. Y. Shi, and W. H. Wang, "Digital subdividing technique for grating signal and its error analysis," Tool Engineering, vol. 40, no. 72-74, 2006.

[14] J. J. Di, Design of High-Precision Displacement Measurement System based on Diffractive Grating, [Ph.D. thesis], Harbin Institute of Technology, China, 2012.

[15] S. F. Liu, Research of Subdivision Technology of Grating Moire Fringe Based on Amplitude Sampling, [Ph.D. thesis], Huazhong University of Science and Technology, China, 2007.

[16] M. J. Lv, Study on Electronic Subdivision of Grating Moiré Fringe, [Ph.D. thesis], Nanjing University of Aeronautics and Astronautics, China, 2008.

[17] K. K. Gai, M. K. Qiu, and H. Zhao, "Energy-aware task assignment for mobile cyber-enabled applications in heterogeneous cloud computing," Journal of Parallel and Distributed Computing, vol. 111, pp. 126-135, 2017.

[18] K. K. Gai, M. K. Qiu, H. Zhao, and X. T. Sun, "Resource Management in Sustainable Cyber-Physical Systems Using Heterogeneous Cloud Computing," IEEE Transactions on Sustainable Computing, no. 99, p. 1, 2017.

[19] C. Roberts, R. L. Johnston, and N. T. Wilson, "A genetic algorithm for the structural optimization of Morse clusters," Theoretical Chemistry Accounts, vol. 104, no. 2, pp. 123-130, 2000.

[20] M. Mitchell, An Introduction to Genetic Algorithms, MIT Press, Cambridge, MA, USA, 1998.

[21] M. K. Qiu, M. Zhong, J. Y. Li, K. K. Gai, and Z. L. Zong, "Phase-change memory optimization for green cloud with genetic algorithm," IEEE Transactions on Computers, vol. 64, no. 12, pp. 3528-3540, 2015.

[22] Z. U. Koreshi and H. Khan, "Optimization of moderator design for explosive detection by thermal neutron activation using a genetic algorithm," Journal of Nuclear Engineering and Radiation Science, vol. 2, no. 3, article 031018, 2016.

[23] M. Srinivas and L. M. Patnaik, "Genetic algorithms: a survey," Computers, vol. 27, no. 6, pp. 17-26, 1994.

[24] G. R. Chorkawy, Exchange Inlet Design Optimization by Genetic Algorithm [Ph.D. thesis], Carleton University, Ottawa, 2011.

[25] D. E. Goldberg, Genetic Algorithm in Search, Optimization, and Machine Learning, Addison-Wesley Pub. Co., Inc., 1989.

[26] J. R. Brauer, "Hall effect and magnetoresistive sensors," Magnetic Actuators and Sensor, pp. 143-163, 2006, Wiley-IEEE Press eBook Chapters.

[27] A. S. Tarnovskii, "Lorentz transformations for electromagnetic field vectors and the motion of charges in constant, homogeneous electromagnetic fields," Soviet Physics Journal, vol. 14, no. 11, pp. 1510-1513, 1971.

[28] T. Kmecl and P. Dalbert, "Investigation of the influence of geometry parameters on performance of a diffuser," International Gas Turbine \& Aeroengine Congress \& Exhibition, pp. 1-11, 1999.

[29] ISO 230-2:2014(E) Test code for machine tools - Part 2: Determination of accuracy and repeatability of positioning of numerically controlled axes. 


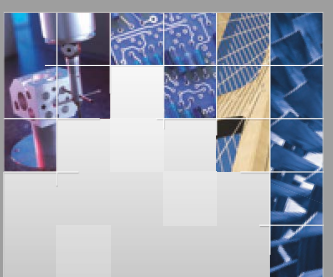

\section{Enfincering}
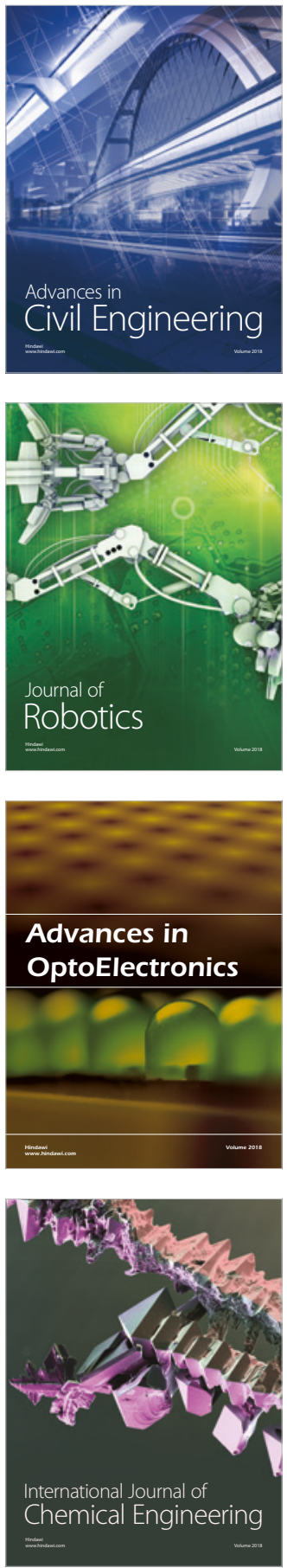

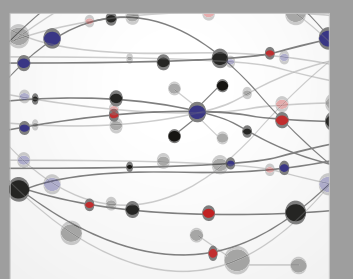

\section{Rotating \\ Machinery}

The Scientific World Journal

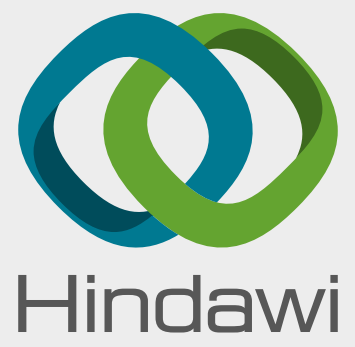

Submit your manuscripts at

www.hindawi.com
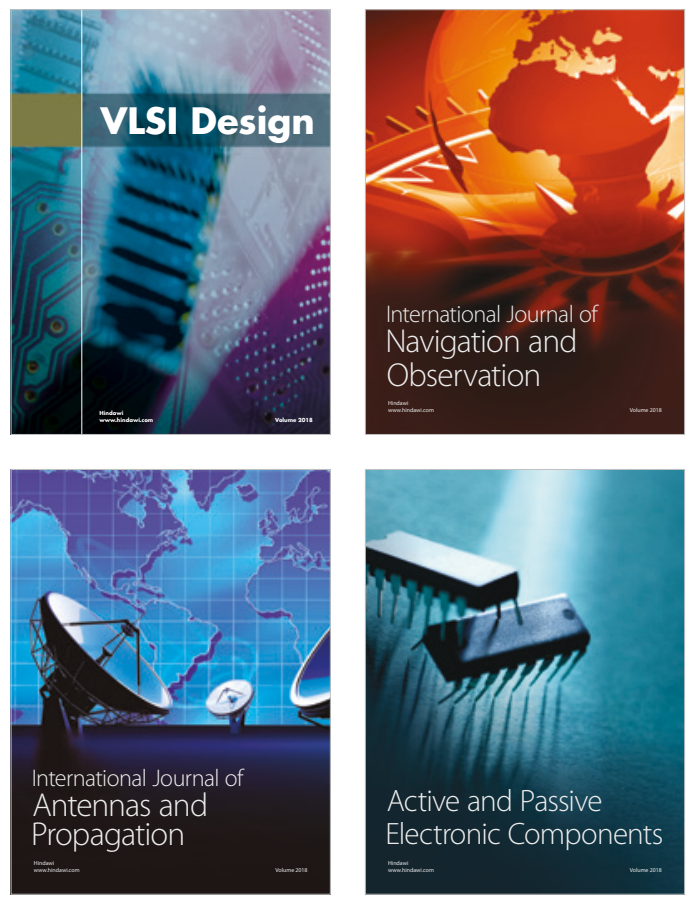
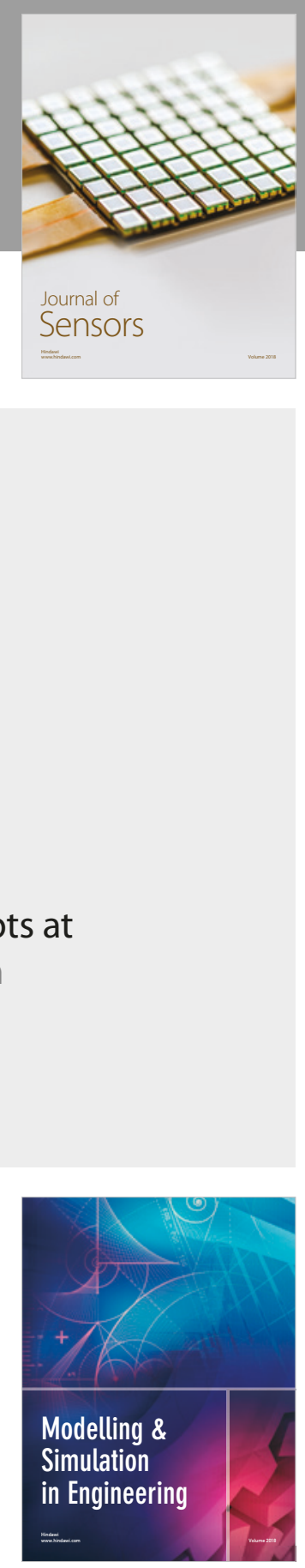

\section{Advances \\ Multimedia}
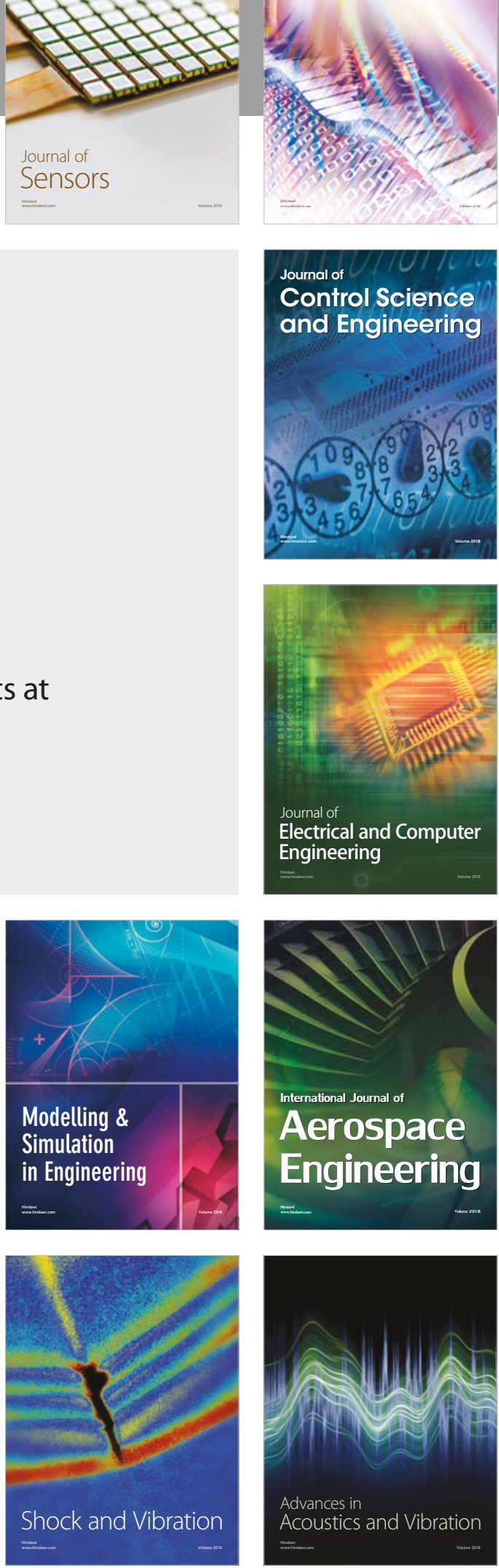